\title{
Emotional Intelligence and Psychopathology in Schizophrenia
}

\author{
Pushpanjali Vishwakarma ${ }^{1 *}$, Dr. Anshul Kumar²
}

\section{ABSTRACT}

Deficits in social cognition, emotion perception and thinking have been extensively documented in schizophrenia either during the course or even in prodromal phase and are associated with poor psychosocial functioning. The present study aimed to explore the associations among emotional intelligence and positive and negative syndromes in the patients with schizophrenia. A sample of 30 male patients with schizophrenia within age range of 18-45 years was taken. Patients were selected by purposive sampling on the basis of inclusion and exclusion criteria from the inpatient department of a psychiatry Institute. PANSS and MSREIS-R were administered on the patients to assess positive, negative symptoms and general symptoms and different components of emotional intelligence respectively. The results revealed that two components of emotional intelligence (a) ability to manage emotions in others (b) total score of MSREIS-R were significantly associated with negative and general symptoms.

Keywords: Schizophrenia, Emotional intelligence, Positive and Negative symptoms

Schizophrenia is a chronic, severe and debilitating psychiatric illness, with a course encompassing a prodromal phase, an active phase, and a residual phase. The active phase has symptoms like hallucination, delusion, and disorganized thinking, called positive symptoms. The prodromal and residual phases are characterized by magical thinking and odd beliefs, as well as deficit in interpersonal relation and self-care; in addition, negative symptoms are alogia (poverty of speech), anhedonia-asociality, affective flattening, avolition-apathy, Psychopathology that involves cognition, emotion, perception, social functioning and other aspect of behavior. (Sadock \& Sadock, 2010). The disorder usually begins before age 25, persists throughout life (Sadock \& Sadock, 2007). Kraepelin (1921) differentiated the major psychiatric illnesses into two clinical types, Dementia precox and manic depressive illness in 1896. Under dementia precox, he brought together the various psychiatric illnesses (paranoia, catatonia and hebephrenic) which were earlier thought to be the distinct illnesses. The emphasis in diagnosis of dementia precox

\footnotetext{
${ }^{1}$ M.Phil Clinical Psychologist at Samarth Psychotherapy and Counseling Center, Indore, M.P., India

${ }^{2}$ Senior Resident Department of Psychiatry, K.D Medical College Hospital and Research Center, Mathura, U.P., India

*Responding Author

(C) 2016, P Vishwakarma, A Kumar; licensee IJIP. This is an Open Access Research distributed under the terms of the Creative Commons Attribution License (http://creativecommons.org/licenses/by/2.0), which permits unrestricted use, distribution, and reproduction in any Medium, provided the original work is properly cited.
} 


\section{Emotional Intelligence and Psychopathology in Schizophrenia}

was on an early onset and a poor outcome (dementia: deterioration; precox: early onset. Bleuler (1950), while renamed dementia precox as a schizophrenia (meaning mental splitting), recognized that this disorder did not always has a poor prognosis as described by Kraepelin. He also recognized that schizophrenia consisted of a group of disorders rather than a distinct entity. Therefore, he used the term a group of schizophrenia. Bleuler (1950), described the characteristic symptoms (fundamental symptoms) which were then thought to be diagnostic of schizophrenia such as ambivalence, autism, affect disturbances, association disturbances. He also described accessory symptoms of schizophrenia (thought to be secondary to fundamental symptoms). These accessory symptoms included delusion, hallucination and negativism. Schneider (1959) described symptoms which, though not specific of schizophrenia, were a great help in making a clinical diagnosis of schizophrenia. These are popularly called as Schneider's first rank symptoms of schizophrenia. He also described the second rank symptoms of schizophrenia which are considered less important for diagnosis of schizophrenia.

Salovey and Mayer (1990) were the first researchers who create the term "emotional Intelligence" in 1990. They used the term Emotional intelligence as "A form of intelligence that involves the ability to monitor one's own and other's feeling and emotions, to discriminate among them and to use this information to guide one's thinking and actions". In this definition of EI, they identified three components of EI e.g. an ability appraise others emotion, an ability to regulate one's own emotion, and ability to use to solve problems. Mayer and Salovey (1997) revised the definition of emotional intelligence as "The ability to perceive emotion, integrate emotion to facilitate thought, understand emotions, and to regulate emotions to promote personal growth."

Patients with schizophrenia experience difficulties in social cognition, emotion, perception and thinking either during the course or even in prodromal phase such as odds beliefs and magical thinking as well as deficits in self-care and interpersonal relationship (Dawsons et al., 2012). Social cognition encompasses the concept of emotional intelligence and is defined as the ability to identify, understand, use and manage emotions, and furthermore, deficit in emotion perception have been associated with poor psychosocial functioning in people with schizophrenia (kee et al., 2009; Ellen et al., 2008). Lack of social cognition may also be a risk factor for psychosis such as poor performance on social cognition tasks was significantly correlated with psychosis in young people who were at ultra-high risk of psychosis (Kim et al. 2011). Other studies indicate that impaired social cognition is significantly associated with schizophrenia. Impairments have been documented in several domains including personal decision making, handling stress lack of empathy, self discloser, self acceptance, insight, affect recognition (Edwards et al., 2002; Fiszdon \& Bell., 2009; Hoekert et al., 2007; Pinkham et al., 2007). However, little is known about other aspects of emotion processing that are critical for adaptive functioning. Several researchers studied the importance and the application of emotional intelligence in schizophrenia. A lower level of emotional intelligence has a significant association with 


\section{Emotional Intelligence and Psychopathology in Schizophrenia}

schizophrenia on all measures of $\mathrm{El}$ and demonstrates poorer neuro-cognition and social functioning (Dawsonet et al., 2012).

The studies that examined the MSCEIT in 50 schizophrenia and 39 non psychiatric controls found that deficits on three of the four subtests which includes identifying, understanding and managing emotions compared to healthy controls (Kee et al., 2009).

Kerns et al. (2008) compared the symptoms profile of 32 patents with disorganized schizophrenia with 34 control participants on characteristics associated with disorganization symptoms in schizophrenia they found that disorganized schizophrenia associated with communication disturbances (CD) than control participants for negative topics but not for positive topics and disorganization symptoms and disorganized schizophrenia both are associated with poor emotion processing.

\section{Rationale for the Study}

Disturbances in social cognition and emotion processing are an important element of the features of schizophrenia. Social cognition encompasses the concept of emotional intelligence, and furthermore, deficits in emotion perception and processing have been extensively documented in schizophrenia and have also been associated with poor psychosocial functioning (Kee et al., 2009, Ellen et al., 2007). Thus, it can be hypothesized that impaired psychosocial and adaptive functioning, as a reflection of impaired emotional intelligence, should also be present in severity of psychopathology like Positive symptoms, Negative symptoms and general symptoms is assumed to be reflected in the profile. Therefore, present study seeks to explore the association among Emotional Intelligence and psychopathology.

\section{METHOD}

Aim

1. To study the association between Emotional Intelligence and psychopathology in persons with schizophrenia.

\section{Objectives}

2. To determine the association between emotional intelligence and positive and negative symptoms in persons with schizophrenia.

The study was conducted at Institute of Mental Health \& Hospital, Agra. Study Protocol was approved by ethical \& scientific committee of the Institute of Mental Health \& Hospital, Agra. Sample consisted of 30 male patients between 18 to 45 years of age with diagnosis of schizophrenia as per ICD-10 Diagnostic criteria. Following tools were administered (i) Sociodemographic and clinical data sheet It includes information like age, sex, education, religion, marital status, occupation, socio economic status etc. The clinical data sheet includes information like duration of illness, age of onset past history, treatment history, history of alcohol and 


\section{Emotional Intelligence and Psychopathology in Schizophrenia}

substance abuse, family history of mental illness, history of significant head injury, seizures and any other significant physical, organic or psychiatric illness, diagnosis.

(ii) Positive and negative syndrome scale (PANSS), developed as remedy for perceived deficits in the brief psychiatric rating scale by Kay et al. (1987). The PANSS includes 30 items on three sub scale, seven item covering positive symptoms and seven item negative symptoms, sixteen item for general psychopathology. Each item is score seven point likert scale ranging from 1-7 (1=absent, 2=minimal, 3=mild, 4=moderate, 5=moderate to severe, 6=severe and 7=Extensive) and score range from 30 (no symptoms) to 210 (very severe psychopathology). An overall score more than 60 indicates a clinically significant condition. Alpha coefficient analysis indicates high internal reliability and homogeneity among items with coefficient ranging from .73 to .83 for each of the scale.

(iii) Multidimensional Self-Report Emotional Intelligence Scale-Revise (MSREIS-R) MSREISR test developed by Panday and Anand,(2008) for the purpose assessing emotional intelligence in five different dimensions- ability to express and appraise emotion, ability to utilized emotion, ability to manage emotion in self, ability to manage emotion in others. This is a likert scale score arrange 1-6 like strongly disagree, averagely disagree, somehow disagree, somehow agree, averagely agree, and strongly agree. Minimum score will be 51 and maximum will be 306 . Full scale reliability Cronbach's alpha coefficient is .95.

\section{Procedure}

Study was conducted at IMHH Agra, after clearance from ethical \& scientific committee. The patients were chosen who already diagnosed schizophrenia according to ICD-10 criteria. After the consent and rapport with subject, the clinical and socio-demographic data was collected. A semi structured socio demographic sheet was use for recording the data. The PANSS was administered for understanding the severity of positive, negative and general symptoms of individual, in last Emotional intelligence scale was administered for getting their score of emotional intelligence.

\section{Data Analysis}

Mean, SD and correlation analysis were done using SPSS 16.0.

\section{RESULT}

The present study was a cross sectional study of 30 subjects with schizophrenia. Mean, SD and correlation analysis were done using SPSS 16.0. The level of significance (alpha) of 0.05 and 0.01 were adopted in the study. These are the tables showing the results of the study below: 
Table 1: Socio-Demographic and Clinical Characteristic

\begin{tabular}{|c|c|c|c|}
\hline \multicolumn{2}{|c|}{ DEMOGRAPHIC VARIABLES } & \multicolumn{2}{|c|}{$\begin{array}{l}\text { PATIENTS WITH SCHIZOPHRENIA } \\
(\mathrm{N}=30)\end{array}$} \\
\hline \multicolumn{2}{|c|}{ Age (in Years) Mean \pm S.D. } & \multicolumn{2}{|l|}{$34.60 \pm 6.85$} \\
\hline \multicolumn{2}{|c|}{ Age of onset of illness (in years) Mean \pm S.D. } & \multicolumn{2}{|l|}{$26.70 \pm 6.59$} \\
\hline \multicolumn{2}{|c|}{ Duration of illness (in years) Mean \pm S.D. } & \multicolumn{2}{|l|}{$7.27 \pm 5.03$} \\
\hline & & $\mathbf{N}$ & $\%$ \\
\hline \multirow{2}{*}{ Marital status } & Married & 18 & $60.0 \%$ \\
\hline & Unmarried & 12 & $40.0 \%$ \\
\hline \multirow{2}{*}{ Religion } & Hindu & 28 & $93.3 \%$ \\
\hline & Muslim & 2 & $6.7 \%$ \\
\hline \multirow{4}{*}{ Education } & Primary & 2 & $6.7 \%$ \\
\hline & Middle & 15 & $50 \%$ \\
\hline & $\begin{array}{l}\text { High school and } \\
\text { Intermediate }\end{array}$ & 9 & $30 \%$ \\
\hline & Graduate & 4 & $13.3 \%$ \\
\hline \multirow{2}{*}{ Occupation } & Employed & 7 & $23.3 \%$ \\
\hline & Unemployed & 23 & $76.7 \%$ \\
\hline \multirow{4}{*}{$\begin{array}{l}\text { Socio-economic } \\
\text { Status }\end{array}$} & Lower & 10 & $33.3 \%$ \\
\hline & Upper lower & 14 & $46.7 \%$ \\
\hline & Middle & 4 & $13.3 \%$ \\
\hline & Upper middle & 2 & $6.7 \%$ \\
\hline \multirow{2}{*}{ Habitat } & Rural & 16 & $53.3 \%$ \\
\hline & Urban & 14 & $46.7 \%$ \\
\hline
\end{tabular}

Table 1: Elaborates different socio-demographic variables including marital status, religion, education, occupation, socio-economic status and habitat. Sample included 18(60\%) married and 12(40\%) unmarried patients among whom $28(93.90 \%)$ of patients belong to Hindu religion while only 2(6.7\%) are Muslims and 16(53.3\%) patients are living in rural areas. 2(6.7\%) of patients are educated up to Primary level, 15(50\%) up to Middle, 9 (30\%) up to High school or Intermediate and only 4(13.3\%) patients are educated up to Graduation. Most of the sample $(\mathrm{N}=23,76.7 \%)$ is unemployed. $10(33.3 \%)$ patients belong to low socio-economic status, 14 (46.7\%) to upper lower, $4(13.3 \%)$ to middle, and only 2 (6.7 \%) belong to upper middle economics status. Mean and standard deviation of the continuous variable like, age, age of onset, and duration of illness were determined. The mean age of the sample is 34.60 with a standard deviation of 6.85. Minimum age of onset of the sample is 20.11 with maximum age of onset being 33.29. Duration of illness varies from 2.23 to 12.31 . 
Table 2 : Correlation Cofficients Between Scores Of Msreis-R Test And Positive And Negative Syndrome Scale Scores

\begin{tabular}{|c|c|c|c|c|c|}
\hline & $\begin{array}{lr}\text { Ability } & \text { to } \\
\text { express } & \text { and } \\
\text { appraise } & \\
\text { emotions } & \end{array}$ & $\begin{array}{l}\text { Ability to } \\
\text { utilize } \\
\text { emotions }\end{array}$ & $\begin{array}{l}\text { Ability to } \\
\text { manage } \\
\text { emotions in } \\
\text { self }\end{array}$ & $\begin{array}{l}\text { Ability to } \\
\text { manage } \\
\text { emotions in } \\
\text { other }\end{array}$ & $\begin{array}{ll}\text { EI } & \text { Total } \\
\text { score } & \end{array}$ \\
\hline $\begin{array}{l}\text { Positive } \\
\text { symptoms }\end{array}$ & -.164 & .078 & .099 & .078 & .005 \\
\hline $\begin{array}{l}\text { Negative } \\
\text { symptoms }\end{array}$ & .004 & -.329 & -.068 & $-.462 *$ & -.226 \\
\hline $\begin{array}{l}\text { General } \\
\text { symptoms }\end{array}$ & -.257 & -.319 & -.116 & $-.461 *$ & $-.363^{*}$ \\
\hline$* \mathbf{p}<0.05$ & & & & & \\
\hline
\end{tabular}

Table 2: Shows bivariate correlation between PANSS scores and Multidimensional Self-Report Emotional Intelligence Scale-R Test scores (MSREIS-R). No statistically Significant relationship is found between positive symptoms and any of dimensions of emotional intelligence on MSREIS-R, while significant negative correlation was found between negative symptoms with the ability to manage emotions in other $(\mathrm{p}<0.05, \mathrm{r}=-.462)$. Ability to manage emotions in other was also found negatively correlated with the general symptoms of schizophrenia at significant level $(\mathrm{p}<0.05, \mathrm{r}=-.461)$. Besides that, total emotional intelligence score on MSREIS-R test was negatively correlated with general psychopathology $(\mathrm{p}<0.05, \mathrm{r}=-.363)$. No statistically significant relationship was found between PANSS scores with the ability to express or appraise emotions, or with the ability to utilize emotions.

\section{DISCUSSION}

The present study was conducted in the Institute of Mental Health and Hospital, Agra with the purpose of exploring the relationship among emotional intelligence and psychopathology. It was a cross sectional correlation study in which subject were selected by using purposive sampling technique based on inclusion and exclusion criteria. Sample consisted of 30 patients with schizophrenia those were already diagnosed by consultant psychiatrist using ICD-10 diagnostic criteria (WHO, 1993). Emotional intelligence was measured by using Multidimensional SelfReport Emotional intelligence Scale-Revised. Positive and Negative Syndrome Scale was used to find the scores on positive symptoms, negative symptoms and general psychopathology.

Findings in the present study revealed no significant correlation between positive symptoms (as measured by PANSS) and the scores on different dimensions of emotional intelligence (as measured by MSREI-R). This was in contrast to the findings observed by Kee et al. (2009) which revealed that in patients with schizophrenia, Identifying Emotions was negatively 


\section{Emotional Intelligence and Psychopathology in Schizophrenia}

correlated with bizarre behavior, Using Emotions with the SAPS total score, and Understanding Emotions with thought disorders and the SAPS total score. The magnitudes of these correlations were small to medium.

In the present study, negative symptoms on PANSS were found negatively correlated with the ability to manage emotions in other but not with the other components like ability to express and appraise emotions, ability to utilized emotions, or with the ability to manage emotions in self as assessed by MSREI-R test. However in the study by Kee et al. (2009) all of the MSCEIT components were having small to medium magnitude of negative correlation with different symptoms of SANS. Sweet et al. (1998) also reported significant relationship between emotional intelligence processes and other negative symptoms measures, including avolition- apathy and anhedonia-asociality.

General symptoms score were correlated negatively with ability to manage emotions in other and with the full scale score of MSREIS-R test in this study. Theses result indicates that various general and negative symptoms (like, unusual thought content, disorientation, depression, guilt feeling, disturbance of volition, active social avoidance, lack of judgment \& insight, difficulty in abstract thinking, blunted affect, emotional withdrawal, apathy, unusual thought etc.) significantly contribute to low emotional intelligence in schizophrenia and that may be the cause why patients with schizophrenia experience difficulties in social functioning, self-awareness, interpersonal relationships, emotional self-control, and self-confidence. In addition, negative symptomatology and emotional intelligence are key components of poor functional outcome in schizophrenia. Thus, the current findings support association between the emotional intelligence assessed by the MSREI-R and several aspects of clinical symptoms but excluding positive symptoms (hallucination, delusion, conceptual disorganization).

\section{CONCLUSION}

The primary aim of the study was to explore associations in emotional intelligence and psychopathology in persons with schizophrenia. The findings revealed that two components of emotional intelligence (a) ability to manage emotions in others (b) total score of MSREIS-R were significantly associated with negative and general symptoms. Deficits in emotional intelligence may either have contributed to or have accumulative effect on the underlying negative and some of general symptoms such as apathy and social avoidance etc in schizophrenia that needs to be investigated through prospective studies. Deficits in emotional intelligence could be predisposing factors for psychiatric illnesses. Emotional intelligence had no association with positive symptoms. This lack of association with positive psychopathology suggests that emotional intelligence is associated more with deficits symptoms than with the positive symptoms in schizophrenia. 


\section{Emotional Intelligence and Psychopathology in Schizophrenia}

Some limitations of the current study should be noted. One of the major limitations of the current study was its cross sectional study design. Second, the female population was not represented in the study which makes it difficult to generalize the result on female population. Third, Purposive sampling might have yielded in selection bias. Fourth, the study population was limited to the patients seeking services in IMHH, Agra. Future studies can also examine if the level of psychopathology can be modified with treatment interventions or if it changes with the course of the illness over the time, how these changes relates to emotional intelligence. Similarly the impairments in emotional intelligence can be tracked in long term including recovery phase.

\section{Acknowledgments}

The author appreciates all those who participated in the study and helped to facilitate the research process.

\section{Conflict of Interests}

The author declared no conflict of interests.

\section{REFERENCES}

Bleuler, E. (1950). Dementia Preccox: The group of schizophrenia. International universities press. New York.

Dawson, S., Kettler, L., Burton, C., Galletly, C. (2012). Do people with schizophrenia lack emotional intelligence? Schizophrenia Research and Treatment, 495174.

Edwards, J., Jackson, H.J., \& Pattison, P.E. (2002). Emotion recognition via facial expression=and affective prosody in schizophrenia: a methodological review. Clinical Psychological Review, 22, (6): 789-832.

Ellen, S.H., \& Woojin, S. (2008). What aspects of emotional functioning are impaired in schizophrenia? Schizophrenia Research, 98, (1-3): 239-246.

Fiszdon, J.M., \& Bell, M.D. (2009). Effects of presentation modality and valence on affect recognition performance in schizophrenia and healthy controls. Psychiatry Research, 170:114-118.

Hoekert, M., Kahn, R.S., Pijnenborg, M., Aleman, A. (2007). Impaired recognition and expression of emotional prosody in schizophrenia: review and meta-analysis. Schizophrenia Research, 96:135-145.

Kay, S.R., \& Opler, L.A. (1987). The positive and negative syndrome scale (PANNS) for schizophrenia. Schizophrenia Bulletin, 13, 261-279.

Kee, K.S., Horan, W.P., Solovery, P., Kern, R.S., Sergi M. J., Fiske, A. P., ..., Green, M.F.(2009). Emotion intelligence in schizophrenia. Schizophrenia Research, 107, 61-69

Kerns, J.G., \& Becker, T.M. (2008). Communication disturbances, working memory, and emotion in people with elevated disorganized schizotypy. Schizophrenia Research, 100(1-3): 172-180. 


\section{Emotional Intelligence and Psychopathology in Schizophrenia}

Kim, S.H., Shin, Y.N., Jang, H.J., Kim, E., Shim, G., Park, Y.H., ..., Kwon, S.J. (2011). Social cognition and neurocogniton as predictors of conversion to psychosis in individuals at ultra-high risk. Schizophrenia Research, 130(1): 170-175.

Kraepelin, E. (1921). Manic-Depressive Insanity and Paranoia. Livingstone. Edinburgh.

Mayer, J. D., \& Salovey, P. (1997). What is emotional intelligence? In P. Salovey \& D. Sluyter (Eds.), Emotional development and emotional intelligence: Implications for educators (331). New York: Basic Books.

Pandey, R., \& Anand, J. (2008). Manual for multidimensional self-report emotional intelligence scale- revised. Varanasi: Rupa psychological center.

Pinkham, A.E., Gur, R.E., \& Gur, R.C. (2007). Affect recognition deficits in schizophrenia: neural substrates and psychopharmacological implications. Expert Review of Neurotherapeutics, 7, 807-816.

Sadock, B.J., \& Sadock, V.A. (2007). Synopsis of Psychiatry: Behavioral sciences/clinical psychiatry (10th ed.). Philadelphia: Lippincott Williams \& Wilkins; 19106-3621.

Sadock, B.J., \& Sadock, V.A. (2010). Pocket Handbook of Clinical Psychiatry (5th ed.). Philadelphia: Lippincott Williams \& Wilkins; 19106.

Salovey, P., \& Mayer, J. (1990). Emotional intelligence.Imagination, Cognition, and Personality, 9,185-211.

Schneider, K. (1959). Clinical psychopathology(5th edn), (trans. M. W. Hamilton). New York: Grune \& Stratton.

Sweet, L.H., Primeau, M., Fichtner, C.G., Lutz, G., 1998. Dissociation of affect recognition and mood state from blunting in patients with schizophrenia. Psychiatr. Res. 81, 301-308.

World Health Organization. (1989) The ICD-1O classification of mental and behavioural disorders: Clinical descriptions and diagnostic guidelines. World Health Organization; Geneva.

How to cite this article: P Vishwakarma, A Kumar (2016), Emotional Intelligence and Psychopathology in Schizophrenia, International Journal of Indian Psychology, Volume 3, Issue 4, No. 68, ISSN:2348-5396 (e), ISSN:2349-3429 (p), DIP:18.01.199/20160304, ISBN:978-1365-39398-3 